\title{
Methods for Quantitative Analysis of Electron Tomographic Reconstructions of Embedded Extensive Biological Structures
}

\author{
R. Reichelt,* L. Kovacik, **
}

* Institut für Medizinische Physik und Biophysik, Universitätsklinikum, Westfälische-WilhelmsUniversität, Robert-Koch-Str. 31, D-48149 Münster, Germany

** Charles University in Prague, First Faculty of Medicine, Institute of Cellular Biology and Pathology, Albertov 4, 12801 Praha 2, Czech Republic

Electron tomographic reconstruction of organic and inorganic objects having a size up to about 400 $\mathrm{nm}$ became in the last years a well-established powerful microscopic technique to characterize structurally nano-scaled specimens $[1,2]$. However, there is a large variety of structures exceeding at least in one dimension the size of about $400 \mathrm{~nm}$ significantly. Plastic-embedding of those structure and subsequent sectioning opens two avenues for electron tomography: (i) studying by TEM the sections [3], or (ii) studying by SEM the block-face freshly revealed after serial sectioning [4].

The presented study deals with the tomographic reconstruction of structures of the channels and the bacula cavities in the wall of negatively stained Epon-embedded hazel pollen grains (Fig. 1) by TEM using $\sim 100-150 \mathrm{~nm}$ thick sections conventionally cut with an ultramicrotome [5]. The sections were investigated at low temperature $\left(\sim-170{ }^{\circ} \mathrm{C}\right)$ with a Tecnai G2 Polara TEM equipped with a Gatan energy filter GIF 2002 and a 2048 x 2048 Gatan CCD camera, operated at $300 \mathrm{kV}$. Tilt series comprised $\sim 120$ projections in the tilt range $\pm 65^{\circ}$. An estimate of the obtainable resolution of the three-dimensional (3D) reconstructions revealed $6-8 \mathrm{~nm}$.

In order to perform reliable quantitative analysis, the irradiation-induced movements of plastic sections were eliminated by splitting of large areas of interest into smaller sub-areas that partially overlapped. Each of the sub-tomograms related to individual sub-areas was separately aligned by conventional sequential cross-correlation followed by several cycles of matching of projections with re-projections of the reconstructed volumes. Typically three to six cycles of this iterative projection matching were necessary until all detected displacements between the positions of the original projections and the computed re-projections were smaller than 1 pixel $(\sim 1.3 \mathrm{~nm})$. The iterative projection matching method used is a simplified version of the method described in [3] but was more suitable for our specimen due to the distribution of stained material within the sections. It significantly improved the quality of the 3D reconstructions because both the number and the magnitude of the arc-like features emerging from the electron-dense structures observable in planes perpendicular to the tilting axis decreased, while the shape of the channels in the cross-sections approximately perpendicular to the channel axis became more compact (Fig. 2).

Due to the complexity of the spatial arrangement of the bacula cavities, segmentation was performed by thresholding. A correct thresholding level was found by comparison of the thresholded original untilted projection with the untilted re-projections of the reconstructed volume thresholded at increasing intensity levels in overlay images (Fig. 3). After the alignment refinement and thresholding, the individual reconstructed sub-volumes were finally assembled back into a large volume by three-dimensional cross-correlation of the individually reconstructed overlapping portions.

The structures of the channels and the bacula cavities in the wall were quantitatively analyzed in order to estimate their role in the release of allergen proteins from the pollen grains. 
References

[1] J.R. McIntosh, ed. Cellular Electron Microscopy, Academic Press, Amsterdam, 2007.

[2] U. Ziese et al., J. Microsc. 205 (2002) 187-200.

[3] F. Cantele et al., J. Struct. Biol. 158 (2007) 59-70.

[4] J.A.W. Heymann et al., J. Struct. Biol. 155 (2006) 63-73.

[5] L. Kovacik et al., J. Struct. Biol. 166 (2009) 263-271.

[6] This research was supported by (i) the NATO Science Fellowship Programme grant No. sine 20/2002, (ii) the State of North Rhine-Westfalia, (iii) industry grants, and (iv) the grants from the Ministry of Education, Youth and Sports of the Czech Republic MSM0021620806 and LC535. The aid of Prof. Monika Grote and Mrs. Ursula Malkus of the Institut für Medizinische Physik und Biophysik and Dr. Jürgen Plitzko of the Max-Planck-Institut für Biochemie (Martinsried, Germany) is gratefully acknowledged.

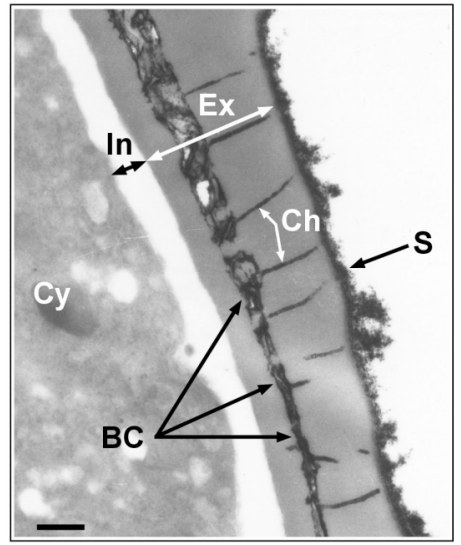

FIG. 1. TEM micrograph of a $80 \mathrm{~nm}$ thick section of a hazel pollen grain, showing the wall with channels $(\mathrm{Ch})$ and bacula cavities (BC). Ex: exine, In: intine, $\mathrm{Cy}$ : cytoplasm, $\mathrm{S}$ : surface. Scale bar: $200 \mathrm{~nm}$.

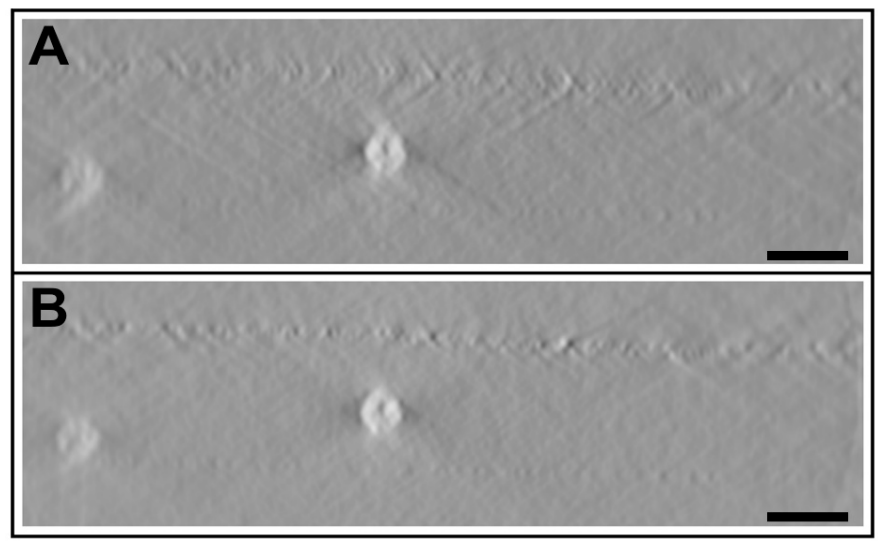

FIG. 2. A selected $\mathrm{x}$-z-cross-section (perpendicular to the tilt axis) through a contrast-inverted sub-volume, which cuts the channels approximately perpendicularly, is shown in (A) after the initial cross-correlation alignment, and in (B) after three loops of the iterative projection matching. Scale bars: $50 \mathrm{~nm}$.

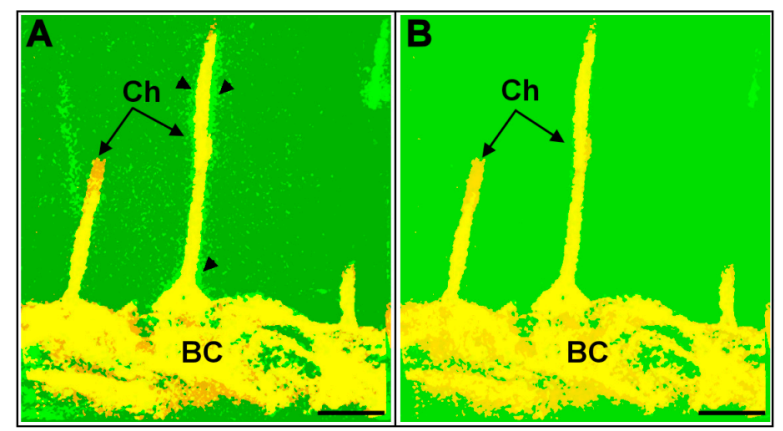

FIG. 3. Estimation of the correct thresholding intensity level in red-green overlay images. The original untilted projection was thresholded according to its bimodal histogram (red image). The reconstructed volume was thresholded at increasing intensity levels and re-projected in the direction of the untilted projection (green image). (A) The threshold level was set too low because the side-arcs around the channels are clearly visible (arrowheads). (B) Re-projection of a correctly thresholded volume contains channels of the same diameter as in the original projection. BC: bacula cavities, Ch: channels. Scale bars: $100 \mathrm{~nm}$. 